\title{
Recht und Religion im islamischen Recht ${ }^{1}$
}

\author{
Von J. Christian Wichard
}

Scharia, islamisches Recht - was ist daran islamisch, was Recht? ${ }^{2}$ Wie funktioniert es zwischen den Anforderungen der Religion auf der einen und den wirtschaftlichen und gesellschaftlichen Bedürfnissen auf der anderen Seite?

Die Schwierigkeiten fangen damit an, daß es das islamische Recht gar nicht gibt, ebensowenig wie es den Islam gibt. Es gibt im islamischen Recht mehrere Schulen, die sich über die Jahrhunderte hinweg entwickelt haben, und auch innerhalb dieser Rechtsschulen gibt es Divergenzen. Ich habe mich im wesentlichen auf zwei Rechtsschulen beschränkt, die hanafitische und die schafi'itische. Beide sind für den sunnitischen Islam repräsentativ. In zeitlicher Hinsicht bleibe ich in der Frühzeit, also der Zeit etwa zwischen 750 und 820 n.C. Das ist die Zeit, in der das islamische Recht seine Prägung erhält, in der die großen Schulautoritäten tätig sind, auf die sich dann die Rechtsschulen berufen. Danach folgt Feinarbeit, das groß angelegte Raster wird ausgearbeitet. Da sich alle späteren Juristen auf die in dieser Zeit gelegten Grundlagen beziehen, hat sie eine besondere Bedeutung, ist also nicht "bloß Geschichte".

Zudem meine ich, daß sich manche der Probleme, das islamische Recht in der heutigen Zeit anzuwenden, besser verstehen lassen, wenn man seine Wurzeln in dieser "formativen Periode" freigelegt und versucht hat, die Bedingungen seiner Entstehung und Entwicklung nachzuvollziehen.

Ich werde in aller Kürze und mit mancher Vereinfachung fünf Aspekte der Differenzierung von Religion und Recht herausarbeiten, die sich - wie ich meine - schon bei den frühen Juristen der hanafitischen und der schafi itischen Rechtsschule finden lassen. Parallel dazu werde ich in drei daran anknüpfenden Exkursen zeigen, wie die Juristen versuchten, religiösen Anspruch und wirtschaftliche Bedürfnisse miteinander zu vereinbaren. Beide

Diese Thematik habe ich ausführlicher am Beispiel des Vertragsrechts behandelt, vgl. Johannes Christian Wichard, Zwischen Markt und Moschee, Wirtschaftliche Bedürfnisse und religiöse Anforderungen im frühen islamischen Vertragsrecht, 1995. Die folgenden Ausführungen bauen auf dieser Untersuchung auf. Dort finden sich auch weitere Hinweise auf die arabischen Quellen.

Vgl. zu diesem Thema auch Baber Johansen, Die sündige, gesunde Amme. Moral und gesetzliche Bestimmung (hukm) im islamischen Recht, in: Axel Havemann, Baber Johansen (Hg.), Gegenwart als Geschichte, Islamwissenschaftliche Studien. Fritz Steppat zum fünfundsechzigsten Geburtstag (FS Steppat), 1988, S. 264-288. 
Stränge, also die Ausdifferenzierung eines rechtlichen Bereiches aus dem religiösen und die Antwort auf Probleme der täglichen Wirtschaftspraxis, hängen zusammen.

I.

Das islamische Recht ist ein offenbartes Recht und als solches eigentlich untrennbar mit der Religion verbunden. Das Recht ist auch Religion und die Religion ist auch Recht. Es gibt zunächst keine echte begriffliche Scheidung. Das Recht ist Bestandteil der Religion, und wie die Religion ist es vom Anspruch her universal und allumfassend: Jede Situation hat ihre Regelung.

Dieser Anspruch stößt allerdings an reale Grenzen. Nicht jede Situation ist ausdrücklich in den autoritativen Rechtsquellen behandelt, also im Koran oder den überlieferten Worten und Taten des Propheten. Manche Antworten erschließen sich nicht unmittelbar aus dem Text sondern müssen erst aus ihm abgeleitet oder unter Berufung auf ihn entwickelt werden, etwa durch einen Analogieschluß $\beta^{3}$.

Dazu sind nur Rechtsspezialisten berufen, weil sie die Rechtsquellen kennen, die Methodik der Rechtsfindung beherrschen und ihre Ergebnisse logisch begründen können ${ }^{4}$. Ihre Expertise gewährt einigen Schutz vor individueller oder kollektiver Willkür. Das ist jedenfalls die Auffassung des juristischen mainstreams, der bis heute herrschenden Meinung.

Halten wir als ersten Aspekt einer Differenzierung zwischen einem "rechtlichen" und einem "religiösen" Bereich fest: Einem unmittelbar einsichtigen Bereich der gesicherten Offenbarung, an dem jeder Gläubige in seiner konkreten Situation teilhaben kann (wenn er in die offenbarten Texte schaut), steht der Bereich der abgeleiteten Rechtsfindung gegenüber, in dem sich nur Spezialisten bewegen können.

II.

Was die Rechtsgelehrten im Wege der Deduktion herausgefunden haben, ist aber nicht die "absolute Wahrheit", es ist nur "äußerlich richtig", hat nur den Anschein von Richtigkeit.

as-Safici (st. 820 n.C.), ar-Risala, hrsg. v. Ahmad Muhammad Sakir, 1358 A.H. (1940 n.C.), S. 477: "Zu allem, was einem Muslim (in seinem Leben) begegnen kann, gibt es eine bindende Anweisung oder einen Hinweis auf den rechten Weg. Wenn es zu dem konkreten Fall eine eigene Regel gibt, so muß sie befolgt werden; gibt es keine, so muß man einen Hinweis auf den rechten Weg mit Hilfe des igtihad suchen, und igtihad ist der Analogieschluß"; vgl. Wichard, a.a.O. S. $62 \mathrm{ff}$.

4 as-Safi $i$, a.a.O. S. 504, $506 \mathrm{ff}$. 
Andere Juristen können durch pflichtgemäße Anstrengung zu einem anderen Ergebnis kommen. Für jeden Juristen, der sich um die richtige Ableitung bemüht hat, ist sein Ergebnis "richtig", und er handelt pflichtgemäß, wenn er sich daran hält, bzw. dementsprechend entscheidet. Als Beleg wird ein Prophetenwort zitiert:

Wenn ein Richter (hakim) durch eigene Überlegung (igtihad) die richtige Entscheidung fällt, so wird er (im Jenseits) doppelt belohnt; irrt er, so wird er nur einfach belohnt ${ }^{5}$.

Damit kommen wir zu dem zweiten Aspekt der Differenzierung: Der unmittelbar einsichtige Bereich hat an der absoluten, unveränderlichen und unumstößlichen Wahrheit der Offenbarung teil. Der Wahrheitsanspruch der abgeleiteten Rechtsfindung reicht dagegen nicht weiter als die begrenzte menschliche Erkenntnisfähigkeit, er ist relativ, "wahr" nur für den jeweiligen Juristen.

Das offenbarte Recht erhebt den Anspruch, abgeschlossen und unveränderlich zu sein. Es steht fest, und es kommen keine neuen Regeln mehr hinzu. Der Statik der Offenbarung tritt die Flexibilität der Ableitung zur Seite: Im relativen Bereich der abgeleiteten Rechtserkenntnis kann es kraft besseren Wissens zu Veränderungen kommen. Diese haben allerdings nur den Charakter von "Korrekturen". Auch für neue, bisher unbekannte Situationen liegen im Recht schon Regelungen bereit, die dann in der konkreten Ableitung erst "entdeckt" werden.

Allerdings ist auch der Bereich der abgeleiteten Rechtsfindung nicht unbeschränkt flexibel. Im Laufe der Jahrhunderte verfestigt sich der überlieferte Bestand des gesicherten juristischen Wissens, die Traditionen der einzelnen Rechtsschulen gewinnen an Gewicht.

\section{Exkurs: Freiheit?}

Versteht man Religion und Recht als vorgegebene, objektive Ordnung, so bleibt wenig Raum für den Begriff der Freiheit im Recht. Die arabischen Worte für "frei" (hurr) oder "Freiheit" (hurriya) drücken ursprünglich einen Status aus, den des unversklavten Menschen, dem Rechte und Pflichten zugeordnet werden können. Ein allgemeiner Freiheitsbegriff hat sich daraus bis in die Neuzeit nicht entwickelt ${ }^{6}$. Das zeigt sich besonders deutlich im Vertragsrecht: Rechtliche Folgen hat eine Handlung nicht deshalb, weil sie gewollt ist, sondern weil ihr diese Folgen in der objektiven Ordnung zugeschrieben werden. Der Ver-

5

6

as-Safi $i$, a.a.O. S. 496; Wichard, a.a.O. S. $65 \mathrm{ff}$.

Franz Rosenthal, The Muslim Concept of Freedom, 1960, S. 11 f, 23 ff.; ders., Artikel "HURRIYYA" in: The Encyclopedia of Islam, 1954 ff., Band 3, S. 589; ders., Artikel "IKHTIYAR", a.a.O. S. 1062. 
trag ist in diesem System das Mittel, Vermögensbestandteile zu genau festgelegten Bedingungen von einem auf einen anderen zu übertragen. Nicht mehr und nicht weniger. Von Vertragsfreiheit ist keine Rede ${ }^{7}$. Das macht es islamischen Juristen auch heute so schwierig, neue Vertragsformen wie etwa den Versicherungsvertrag oder viele der heute üblichen Bankgeschäfte auf dem Boden des islamischen Rechts anzuerkennen ${ }^{8}$. Der Begriff der Vertragsfreiheit setzt ein vertragliches Weltbild voraus, in dem der Vertrag prinzipiell allumfassend ist, ein Vehikel der Emanzipation des Individuums und Grundlage der Ordnung der Gesellschaft. Im islamischen Recht ist diese Ordnung vorgegeben. Auch das Vertragsrecht ist Sache Gottes, eine objektive Ordnung, die nicht zur Disposition des menschlichen Willens steht, sondern ihm den Bereich absteckt, in dem er wirken kann.

Die abgeleitete Rechtsfindung ist nur ein Mittel zur Konkretisierung dieser Ordnung und nur beschränkt zur Fortentwicklung des Rechts tauglich. So hatten die islamischen Juristen der Frühzeit große Probleme mit dem Werklieferungsvertrag (istisna $\left.{ }^{c}\right)^{9}$, der allgemein üblich, jedoch in den autoritativen Texten nirgends erwähnt war. Dabei verpflichtete sich ein Handwerker, ein bestimmtes Werk aus eigenem Material herzustellen. Nach Aufassung der frühen islamischen Juristen kommen Verträge erst dann unter den Schutz des Rechts, wenn sie sich in eines der vorgegebenen Vertragsschemata einordnen lassen, und diese Auffassung wirkt bis heute nach. Der Werklieferungsvertrag ist jedoch kein Kaufvertrag, weil das Vertragsobjekt noch gar nicht existiert. Er paßt auch nicht unter die anderen anerkannten Vertragstypen. Die Juristen behalfen sich damit, daß sie den Vertrag erst dann juristisch faßten, wenn das Objekt fertiggestellt war, wenn also die Situation eines Kaufvertrages gegeben war. Frühestens zu diesem Zeitpunkt war der Vertrag für beide Teile bindend und erzwingbar. Das ist natürlich für den Handwerker sehr nachteilig, weil er das Werk herstellen muß, ohne sicher sein zu können, daß der Besteller es ihm dann auch tatsächlich abnimmt. Diese Art der Rechtsfortbildung wird uns noch häufiger begegnen: Praktiken, die sich nicht in das religiös fundierte Rechtssystem einordnen lassen, werden "maskiert" - hier als Kaufvertrag - und geduldet.

Ausführlicher zur Vertragsf reiheit im islamischen Recht Wichard, a.a.O. S. $231 \mathrm{ff}$.

Einige Materialien zur heutigen islamischen Diskussion über die Versicherung finden sich bei Rüdiger Lohlker, Schari`a und Moderne. Diskussionen über Schwangerschaftsabbruch, Versicherung, Zinsen, 1996, S. 47 ff.; zum Problem "islamischer Bankgeschäfte" vgl. auch Florian Amereller, Hintergründe des "Islamic Banking". Rechtliche Probleme des riba-Verbotes in der Shari`a und seine Auswirkungen auf einzelne Rechtsordnungen arabischer Staaten, 1995; Gerhard Wegen, Johannes Christian Wichard, Islamische Bankgeschäfte, RIW 1995, S. 826-830.

Vgl. dazu Wichard, a.a.O. S. 237 ff. 
III.

Die objektive Ordnung muß durch abgeleitete Rechtsfindung konkretisiert werden. Deren Relativität setzt sich fort in der Unmöglichkeit, im tatsächlichen Bereich die Gewißheit der Wahrheit zu haben.

Die Fähigkeit der Menschen, die entscheidungserheblichen Tatsachen zu ermitteln, ist begrenzt. Das kann zu paradoxen Situationen führen, wie ein Beispiel bei dem Juristen as$S a f i^{c} i$ zeigt: Einen Muslim dürfen und müssen wir als solchen behandeln; wir dürfen ihn also heiraten und erben lassen, und sein Leben ist unantastbar. Selbst dann ist er als Muslim zu behandeln, wenn er tatsächlich im Inneren ungläubig ist. Wer jedoch sicher weiß, daß er es mit einem Apostaten zu tun hat, darf ihn töten, darf ihn nicht heiraten und erben lassen, u.s.w. Trotz des völlig entgegengesetzten Verhaltens handeln sowohl wir als auch die anderen pflichtgemäß ${ }^{10}$. Was richtiges und pflichtgemäßes Handeln ist, bestimmt sich also nicht zuletzt nach dem jeweiligen Wissensstand.

In der Ermittlung der entscheidungserheblichen Tatsachen sind die zur Rechtsanwendung Berufenen auf die Mittel des Prozeßrechts beschränkt: Jedes Rechtssystem schafft sich einen anerkannten Satz von Mitteln, mit denen entscheidungsrelevante Anhaltspunkte rekonstruiert werden können. Diese gelten fortan für das Recht als wahr. Im islamischen Prozeßrecht sind das das Anerkenntnis, der Zeugenbeweis, der Abwehr- und der Bekräftigungseid. Der Richter ist an die Beweisergebnisse gebunden, einen Grundsatz der freien Beweiswürdigung gibt es nicht. As-Safi $i^{i} i$ beschreibt, wie er einen Zivilprozeß entscheidet: Wenn der Beklagte seine Verpflichtung anerkennt, so entscheidet er zu seinen Lasten. Erkennt der Beklagte nicht an, so hält sich $a s-S_{\text {Sai }}{ }^{i}$ als Richter an die Aussage zweier Zeugen. Sind keine Zeugen verfügbar, so legt er dem Beklagten einen Eid auf. Schwört dieser, so wird die Klage abgewiesen. Verweigert er den Eid, so muß sein Gegner, der Kläger, schwören, wenn er mit seinem Verlangen durchdringen möchte. Diese Beweismittel sind nicht alle gleich zuverlässig. Man muß sich aber mit dem jeweils verfügbaren Grad an "Wahrheit" begnügen. Zu mehr ist man vor Gott nicht verpflichtet ${ }^{11}$.

Daran läßt sich das Bemühen erkennen, die richterliche Entscheidung möglichst an objektive Kriterien zu binden und Willkür fernzuhalten (also gerade das Gegenteil von Kadijustiz). Die frühen Juristen heben in diesem Zusammenhang hervor, daß etwa die Strafe beim Ehebruch nur gestützt auf Zeugenaussagen verhängt werden dürfe. Scheitert der Zeugenbeweis, so darf der Richter nicht verurteilen, selbst wenn er - etwa aufgrund

10 as-Safici, a.a.O. S. 482 .

11 as-Safi ${ }^{c}$ i, a.a.O. S. 483; vgl. zum islamischen Prozeßrecht auch Baber Johansen, Le jugement comme preuve. Preuve juridique et vérité religieuse dans le droit islamique hanéfite, Studia Islamica 72 (1990), S. 5-17. 
bestimmter Indizien - etwas anderes argwöhnt. Wer sich von seinem Argwohn leiten läßt und gegen das "Äußere" entscheidet, handelt gegen Koran und Sunna ${ }^{12}$.

Besonders deutlich zeigt sich die Unzulänglichkeit der menschlichen Erkenntnisfähigkeit dann, wenn es darum geht, "innere Tatbestände" zu ergründen, also Motive, Vorstellungen und Absichten der Parteien. Diese sind, sofern sie nicht ausdrücklich erklärt wurden, der rechtlichen Beurteilung nicht zugänglich.

Damit ergibt sich als ein dritter Aspekt der Differenzierung zwischen Recht und Religion: Die rechtliche Beurteilung menschlichen Verhaltens im Diesseits orientiert sich nur am äußeren, objektiv sicher feststellbaren Erscheinungsbild, die religiöse Beurteilung durch Gott im Jenseits bezieht auch Motive, Wünsche und Absichten mit ein, die unerkannt bleiben $^{13}$

Im Vertragsrecht hat das zur Konsequenz, daß ein Vertrag, der äußerlich allen rechtlichen Anforderungen entspricht, nicht deshalb für nichtig erklärt werden darf, weil man dahinter eine "böse Absicht" vermutet, eine Umgehung des Wucherverbots etwa. Solange das äußere Erscheinungsbild unanfechtbar ist, muß der Vertrag als wirksam anerkannt werden. Eine eventuell dahinterstehende Absicht, die als Gegenstand einer rechtlichen Vereinbarung den Vertrag nichtig machen würde, wird mißbilligt, doch beeinflußt die Mißbilligung die rechtliche Wirksamkeit des Vertrages nicht. Ein Beispiel: Wenn jemand eine Frau mit der festen Absicht heiratet, diese sofort nach der ersten Nacht wieder zu verstoßen, so ist der Ehevertrag wirksam, weil er äußerlich fehlerlos ist. Anders wäre es hingegen, wenn die Befristung in den Vertrag selbst mit aufgenommen worden wäre. Da sie dann an der Oberfläche des Rechtsaktes selbst läge, würde sie ihn infizieren, der Vertrag wäre nichtig ${ }^{14}$.

Diese Konsequenz ziehen allerdings nicht alle Juristen in gleichem Maße. Manche sind durchaus bereit, Verträge, die in Umgehung eines rechtlichen Verbotes abgeschlossen wurden, ebenfalls unter das Verbot zu ziehen und für nichtig zu erklären. Andere wiederum dehnen den formalen Grundsatz so weit aus, daß sie aktiv an der Formulierung von Umge- 
hungsgeschäften teilnehmen ${ }^{15}$. Hier kommt es zu sehr interessanten Disputen unter den islamischen Rechtsschulen ${ }^{16}$.

\section{Exkurs: Die Aneignung der Praxis durch das Recht}

Mit Hilfe des Formalcharakters konnten manche im Handel gebräuchlichen Praktiken rechtlich gefaßt werden, die sich nicht bruchlos in das Rechtssystem einordnen ließen. Ein Beispiel dafür, den Werklieferungsvertrag, habe ich oben schon angesprochen. Ein weiteres Beispiel ist die suftaga, ein im Fernhandel bedeutsames Instrument des bargeldlosen Zahlungsverkehrs ${ }^{17}$. Es verbindet Elemente der Forderungsabtretung und der Anweisung: Entweder verpflichtete sich dabei der Schuldner einer Forderung, den geschuldeten Betrag an einem anderen Ort zurückzuzahlen, ggf. auch auf Anweisung seines Gläubigers an einen Dritten, oder der Schuldner wies schriftlich einen dort ansässigen Dritten an, dem Gläubiger den Betrag vor Ort zu erstatten. Die Juristen konnten die suftaga nicht als eigenen Vertragstyp anerkennen, da es dafür keine Grundlage in den Texten gibt. Sie behandelten sie daher nach den Grundsätzen des Darlehens. Beim Darlehen war es allerdings bedenklich, wenn sich der Schuldner verpflichtete, irgend etwas über die bloße Rückzahlung hinaus zu unternehmen, denn beim Darlehen konnte nur die Rückzahlung des empfangenen Betrages vereinbart werden, alles andere unterfiel dem riba-Verbot ${ }^{18}$. Doch solange die Bedingung, daß die Schuld an einem anderen Ort zurückgezahlt werden müsse, nicht ausdrücklich vereinbart war, hatten die Juristen nichts dagegen einzuwenden, wenn die Menschen tatsächlich so verfuhren.

An solchen Beispielen zeigt sich wieder der Kompromißcharakter des islamischen Rechts in der Interpretation der von mir untersuchten Rechtsschulen: Als objektive Ordnung ist es dem menschlichen Willen nicht verfügbar. Gestaltungsspielraum, etwa für die Eingliederung allgemein üblicher Vertragsformen, wird außerhalb, bzw. am Rande dieses Bereiches gewonnen. Verträge, die sich äußerlich im vorgegebenen Rahmen halten, tragen die Vermutung der Wirksamkeit in sich. Abreden im Vorfeld des Vertrages, formlose Verständigungen stören den absoluten Geltungsanspruch der objektiven Ordnung nicht und werden

Ein Produkt solcher Hilfestellungen für die Praxis sind die verschiedenen kutub al-hiyal der hanafitischen Rechtsschule. Vgl. dazu etwa Joseph Schacht, Die arabische hiyal-Literatur, Der Islam 15 (1926), S. 211-232; Wichard, a.a.O. S. $81 \mathrm{ff}$.

Vgl. dazu Wichard, a.a.O. S. 73, 81 ff. und passim; Laoust, Essai sur les doctrines sociales et politiques de Taki-d-Din Ahmad bin Taimiya, 1939, S. 229, 410, 424 ff.; ein Beispiel aus der aktuellen Diskussion über das Verbot von riba ("Wucher") bei Lohlker, a.a.O. S. $133 \mathrm{ff}$.

Vgl. Wichard, a.a.O. S. 247; vgl. auch Armin Remde, Lettera di Cambio und suftadja. Eine rechtsvergleichende Studie zur Entstehungsgeschichte des Wechsels, 1969.

18

Vgl. zum Darlehen, Wichard, a.a.O. S. $201 \mathrm{ff}$. 
geduldet. Auf diese Weise kommt es nicht zu einer offenen Anerkennung und Eingliederung der Praxis in das Recht. Das Recht stellte als objektive, nicht verfügbare Ordnung zu starre Barrieren gegen das Eindringen von Gewohnheitsrecht auf. Um aufgenommen zu werden, mußten Praktiken sich des Gewandes der vorgegebenen Normen bedienen, oft einer Verkleidung, ja Maskierung. Das islamische Recht vor allem der hanafitischen Rechtsschule ist im Anspruch streng, in der Ausführung aber kompromißbereit. In diesem Kompromiß werden religiöser Anspruch und praktische Bedürfnisse nicht miteinander verbunden, sondern letztlich aneinander vorbeigeführt, um Kollisionen zu vermeiden.

IV.

Die Absichten der Menschen richtet Gott, ihre Handlungen können auch die Menschen beurteilen. Hier scheint die Doppelnatur des islamischen Rechts auf: Zum einen ist es eine für den einzelnen gegenüber Gott verbindliche Pflichtenordnung, zum anderen ist es ein formal im Diesseits mit allen weltlichen Beschränkungen durchzusetzendes Recht.

Damit ist der Boden für eine weitere Differenzierung bereitet, die sich bei allen Juristen findet. Im islamischen Recht wird unterschieden zwischen Normen, die auch "im Diesseits" gerichtlich erzwingbar sind und solchen, die nur "im Jenseits" sanktioniert werden ${ }^{19}$. In Anlehnung an Max Weber kann man von einer "äußeren Garantie" durch Gerichte und einer "inneren Garantie" über das verantwortliche Gewissen des Gläubigen sprechen.

Ein Beispiel, das für das Vertragsrecht relevant ist ${ }^{20}$ : Ein bloßes Versprechen bindet den Versprechenden nach Ansicht der meisten Juristen nur in seinem Verhältnis zu Gott. Nur ihm gegenüber muß er sich verantworten, wenn er ein Versprechen nicht hält. Im Diesseits hat ein Versprechen jedoch keine rechtlichen Wirkungen. Derjenige, dem gegenüber es abgegeben wurde, kann daraus nicht klagen. Bindend sind nur Austauschverträge - eine Situation wie im englischen Common Law, das im Vertragsrecht stets eine consideration voraussetzt. Im islamischen Recht kann die Unverbindlichkeit "bloßer Versprechen" wiederum mit Hilfe des Formalcharakters aufgefangen werden: Im Prozeß ist das Anerkenntnis - wie wir gesehen haben - für den Richter verbindlich. Ein solches Anerkenntnis kann aber auch außerhalb eines Prozesses abgegeben werden. Dann muß es im Prozeß ggf. beweisbar sein. Auf diese Weise kann auch durch einseitige Erklärung, das Anerkenntnis, eine bestimmte Rechtslage hergestellt werden. Diese Wirkung beruht jedoch nicht auf einer offenen Anerkennung der verpflichtenden Kraft des Willens, womit der Weg zur Vertrags- 
freiheit bereitet wäre. Sie basiert vielmehr rein formal auf der prozessualen Rolle des Anerkenntnisses.

Ein weiteres Beispiel für die Differenzierung zwischen gerichtlich durchsetzbaren und nur vor dem "forum internum" verpflichtenden Normen bieten die unentgeltlichen Verträge. Sie sind gerichtlich nicht erzwingbar. Es ist zwar verwerflich, eine Schenkung zu widerrufen, rechtlich ist das jedoch grundsätzlich möglich. Nur solche Verträge sind bindend, die auf einen Austausch zurückgeführt werden können. "In unentgeltlichen Geschäften gibt es keinen Rechtszwang" ${ }^{21}$, sagen die späteren Juristen, sie stehen außerhalb des rechtlich geregelten Bereichs. Auch damit ist Raum zur Duldung bestimmter Praktiken gewonnen, die sich nicht in das System des Vertragsrechts einfügen lassen: Was nicht ausdrücklich vereinbart ist, kann nachträglich als "Schenkung" anerkannt werden.

Ein letztes Beispiel für die Differenzierung zwischen gerichtsverbindlichen und Gewissensnormen $^{22}$ : Einige Überlieferungen verbieten gewisse Praktiken, die man heute dem Recht gegen den unlauteren Wettbewerb zuordnen würde: Das Horten von Lebensmitteln etwa, das Abfangen von Karawanen vor dem Markt, um einen besonders günstigen Preis zu erzielen oder das Scheinbieten auf einer Auktion mit dem alleinigen Ziel, den Preis hochzutreiben. Diese Verbote sind gerichtlich nicht durchsetzbar und haben auch sonst keine Rechtsfolgen. Wer gegen sie verstößt, ist ein Sünder. Im Rahmen des Kaufrechts sind solche Verbote rechtlich nicht faßbar, weil es nur den individuellen, zwischen zwei Parteien abgeschlossenen Vertrag betrachtet.

Diese Differenzierung zwischen gerichtsverbindlichen und Gewissensnormen wird von as$\mathrm{Safi}_{i}{ }^{i}$ theoretisch fundierter festgemacht an der Zuordnung von Rechten ${ }^{23}$ : Im Diesseits sanktioniert werden nur Verletzungen von Rechten, die einem anderen unter dem Schutz des Rechts zugeordnet sind. So unterscheidet er zwei Arten von Verboten: Einerseits solche, in denen etwas "an sich" verboten bzw unantastbar und nur aufgrund eines besonderen Hinweises in Koran oder hadit erlaubt ist ("Verbot mit Erlaubnisvorbehalt"), zum Beispiel das Vermögen eines anderen. Das "Objekt" der Handlung, das Vermögen, ist rechtlich einem anderen zugeordnet. Erlaubt werden solche Dinge für andere nur mit den Mitteln und zu den Bedingungen, die Koran und sunna festgelegt haben, das Vermögen also durch den Kaufvertrag, wie er in den autoritativen Texten ausgeformt worden ist. Verstößt jemand gegen die niedergelegten Wirksamkeitsvoraussetzungen, so ist das Vermögen weiter für ihn verboten. 
Auf der anderen Seite stehen nach $a s-S a f i{ }^{i} i$ solche Fälle, in denen etwas grundsätzlich erlaubt und nur unter bestimmten Umständen verboten ist ("Erlaubnis mit Verbotsvorbehalt").

Wo etwas die "rechtliche" Zuordnung eines "Rechtes" zu einem anderen berühren kann, sind Normen gerichtlich erzwingbar; wo nicht, verpflichten sie den Einzelnen nur in seinem Verhältnis zu Gott, die Sanktion erfolgt im Jenseits.

\section{Exkurs: Auseinanderfallen von rechtlicher und religiöser Wertung}

Aus der Differenzierung zwischen gerichtsverbindlichen und Gewissensnormen resultiert ein Problem ${ }^{24}$, das uns auch zuvor schon mehrfach begegnet ist: Religiöse Normen müssen nicht rechtlich erzwingbar sein, rechtliche Normen sollten jedoch von religiösen gedeckt sein und ihnen zumindest nicht widersprechen. Wenn nun aber Recht und Religion in manchen Bereichen eigenen Regeln folgen, kann es passieren, daß beide auseinanderfallen und eine rechtlich unanfechtbare Lage entsteht, die jedoch religiös-moralisch unerträglich ist.

Besonders deutlich werden die Unzulänglichkeiten der menschlichen Rechtsfindung im Prozeß. Hier kann es wegen des strengen Formalcharakters passieren, daß jemandem auf legalem, also formal rechtmäßigem Wege etwas zugesprochen wird, was ihm "eigentlich" nicht zusteht. In solchen Fällen ist das Gewissen des Einzelnen gefordert, das Gefühl der Verantwortung gegenüber Gott, die "innere Garantie", die durch jenseitige Strafdrohungen angespornt wird:

...der Prophet sagte: "Demjenigen, der durch seinen Meineid einem Muslim sein

Recht beschneidet, verweigert Gott das Paradies und bestimmt ihn für das Feuer." ${ }^{25}$

Umgekehrt kann vor dem "religiösen Forum" ausnahmsweise auch ein rechtswidriges Verhalten gerechtfertigt sein: Droht etwa einem zu Unrecht Verklagten, der die wahre Rechtslage nicht beweisen kann, im Prozeß ein Rechtsverlust, so darf er ausnahmsweise einen Eid ablegen, von dem er weiß, daß er den Tatsachen nicht entspricht ${ }^{26}$.

In beiden Fällen weist das islamische Recht weit über die weltlichen Gerichte hinaus.

24

25

26

Vgl. Wichard, a.a.O. S. 77 ff.; Johansen, FS Steppat, S. $273 \mathrm{ff}$.

Malik ibn Anas (st. 795 n.C.), al-Muwatta', Hrsg. v. M. Fu'ad 'Abd al-Baqi, Band 2, S. 727 Nr.11.

Vgl. das Beispiel bei Wichard, a.a.O. S. $83 \mathrm{f}$. 
Anforderungen des Wirtschaftsverkehrs führten dazu, daß das Recht noch in einer weiteren Hinsicht gegenüber der Religion Autonomie gewinnen konnte:

Zumindest das Vermögensrecht gilt nämlich auch für Nichtmuslime ${ }^{27}$. Partner eines Kaufvertrages kann jeder sein, der Eigentümer ist, unabhängig von seiner sozialen oder religiösen Stellung. Damit sind nur die Sklaven ausgeschlossen, die nicht rechtsfähig sind. Das Kaufrecht ist allgemein und gilt für alle freien Untertanen. Ein Zitat aus einem frühen Rechtswerk:

"Die Christen stehen in allen Kaufverträgen den Muslimen gleich, mit Ausnahme von solchen über Wein und Schwein" ${ }^{28}$.

Das Recht ist hier in erster Linie Verkehrsrecht, nicht religiöses Recht. Als Verkehrsrecht ist es auf eine "äußere Garantie", seine gerichtliche Erzwingbarkeit, beschränkt. Anders wäre es auch nicht möglich gewesen, den Güteraustausch juristisch abzusichern, denn der macht nicht an den konfessionellen Grenzen halt. Der religiöse Charakter des Rechts tritt in den Hintergrund und damit auch die "innere" Garantie der Normen über das verantwortliche Gewissen des Gläubigen seinem Gott gegenüber.

VI.

An fünf verschiedenen Linien habe ich versucht, einen "spezifisch rechtlichen" Bereich aus dem Gesamtkorpus des islamischen Rechts herauszutrennen. Das geht nicht ganz chirurgisch, denn völlig abtrennbar ist nichts; alles bleibt vielfältig aufeinander bezogen. Aber den Juristen sind die Unterschiede deutlich: Es ist ein Bereich abgeleiteter, technischer Rechtsfindung, der Spezialisten in die Hand gegeben ist und seine Legitimität auch aus ihrer Expertise bezieht; auf das Diesseits bezogen teilt er alle Beschränkungen des menschlichen Verstandes, innere Sachverhalte sind ihm nicht zugänglich, und der Prozeß kann nur mit einer formalen Wahrheit arbeiten; wesentliches Charakteristikum ist, daß seine Normen gerichtlich erzwingbar und insofern "äußerlich garantiert" sind, und dieser Bereich kann sogar Nichtmuslime erfassen. Hinter allem aber steht eine religiöse Ordnung von Ge- und Verboten, ein Pflichtensystem, das sich an den einzelnen Gläubigen wendet und ihn durch sein Leben lenken soll. Das Recht zu befolgen, ist Gottesdienst. Dadurch bleibt der rechtliche Bereich eng an den religiösen gebunden; nur von ihm erhält er letztlich seine Legitimität.

Wichard, a.a.O. S. 79 f.; Johansen, FS Steppat, S. 269.

28

as-Saibani (st. 805 n.C.), Kitab al-Asl fi 'l-furu', hrsg. v. Chafik Chehata, 1954, S. 221 Nr. 20. 
Das Recht wurde in einem religiös geprägten Umfeld gelebt, in dem die Religion einen viel stärkeren Einfluß auf den Einzelnen ausübte und wo die Einhaltung der "bloß religiösen" Vorschriften ggf. auch gesellschaftliche Sanktionen nach sich ziehen konnte. Natürlich war auch die juristische Infrastruktur nur schwach ausgebildet, es gab kein flächendeckendes Gerichtssystem. Deshalb setzt das klassische islamische Recht die "innere Garantie" der Normbefolgung in viel stärkerem Maße voraus als die meisten unserer heutigen Rechtsordnungen. Auf diese Weise gleicht die Religion Schwächen des Rechts aus. Dadurch verlagert sich ein großer Teil der Verantwortung für die korrekte Einhaltung der Vorschriften auf den Einzelnen und sein Gewissen. Dabei ermöglicht es das Zusammenspiel von religiöser und rechtlicher Garantie, übergroße Härten und allzu formale Regeln abzumildern. Das gestattet den Kompromiß zwischen Vorschrift und Gewissen.

Dieser Kompromißcharakter prägt auch die Einstellung der von mir untersuchten Rechtsschulen gegenüber den Bedürfnissen der Praxis. Die Praxis muß sich der vorgegebenen objektiven Ordnung unterordnen, sie wird nicht offen als rechtsschöpferischer Faktor anerkannt. Gestaltungsspielraum wird im Vorfeld, am Rande des rechtlich geregelten Bereiches gewonnen (Anerkenntnis, unentgeltliche Verträge). Dabei geben die Juristen durchaus auch Hilfestellung. Äußerlich bleibt der vorgegebene Bereich der "objektiven Rechtsordnung" unangetastet. Dennoch geht die Entwicklung neuer Vehikel des Parteiwillens letztlich zu seinen Lasten.

Läßt sich aus all dem etwas für die heutigen Probleme des islamischen Rechts ableiten? Es ist schwer, das ohne starke Vergröberungen zu tun. Einerseits erschwert der religiöse Anspruch eine Fortentwicklung der rechtlichen Bestimmungen. Andererseits ist er aber auch kaum von ihnen zu trennen: Viele der Rechtsvorschriften, um deren Anwendung als Zeichen der Re-Islamisierung es heute in vielen Staaten der islamischen Welt geht, wirken ohne die Einbettung in die alles überwölbende Pflichtenordnung übermäßig rigide und formalistisch. Wird das Recht der vielfältigen Voraussetzungen entkleidet, die es umgeben, so bleibt nur ein furchterregendes Gerippe starrer Normen. 


\title{
The Relationship between Law and Religion in Islamic Law
}

\author{
By J. Christian Wichard
}

Shari'a, Islamic law, is closely linked to law and to religion. However, the first question to be answered is, what is Islamic law? After a first introduction to specific traits of Shari'a, five examples show different legal aspects of Islamic law as a whole.

The first aspect discussed is that Islamic law is revealed law which leads to an inseparable link between law and religion - with few exceptions. As a second aspect, it has to be noted that the deduction of law by lawyers does not lead to an absolute result, or absolute truth, but is only of relative relevance to the specific decision. The relativity of law and the limits to human fact and law finding leads to a demand for objective criteria to support a legal decision and to avoid arbitrary verdicts, one of the reasons of the greater importance attributed to external facts as compared to motives and intentions. Fourth, the fact that man can only decide on objective facts, but not internal motives, leads to a differentiation between norms sanctioned here and others sanctioned "hereafter". The fifth aspect pointed out is the equality between Christians and Muslims in some areas of the law of contracts. The author concludes, inter alia, that although the given objective order is not touched there is room for new solutions along the fringes - outside the normative order. On the one hand, this shows how the development of law is slowed down by the religious link, on the other hand, Shari'a is misunderstood to be much more strict, if separated from its religious background, which is the basis for its understanding.

\section{Conservation of Nature by International Law and Sustainable Development in Overseas Countries}

\section{By Ulf Marzik}

The notion of sustainable development, which has been introduced by the World Conservation Strategy of 1980, may be considered one of the key expressions in international relations and, especially, in international environmental law. It addresses one of the most pressing issues the international community is facing today: the conflict between the protection of the environment and the use of natural resources for further development.

The 1992 Convention on Biological Diversity is the first global convention on the conservation of nature to include the concept of sustainable development in its provisions. Primarily, the convention is focussing on a specific aspect of nature conservation, the protection of biological diversity, and is thus promoting the conservation of nature by international law to meet this newly acknowledged threat to the ecosystem. The article, therefore, outlines the development of nature conservation by international treaties from 